“C 2019 IEEE. Personal use of this material is permitted. Permission from IEEE must be obtained for all other uses, in any current or future media, including reprinting/republishing this material for advertising or promotional purposes, creating new collective works, for resale or redistribution to servers or lists, or reuse of any copyrighted component of this work in other works." 


\title{
Analog Least Mean Square Loop for Self-Interference Cancellation: Implementation and Measurements
}

\author{
Anh Tuyen Le ${ }^{1}$, Le Chung Tran ${ }^{2}$, Xiaojing Huang ${ }^{1}$, and Y. Jay Guo ${ }^{1}$ \\ ${ }^{1}$ University of Technology Sydney, Ultimo, NSW, 2007, Australia \\ Email: \{anhtuyen.leestudent.uts.edu.au \\ Email: \{xiaojing.huang, jay.guo\}euts.edu.au \\ ${ }^{2}$ University of Wollongong, Wollongong, NSW, 2522, Australia \\ Email: lctran@uow.edu.au
}

\begin{abstract}
Analog least mean square (ALMS) loop is a simple and efficient adaptive filter to cancel self-interference (SI) in inband full-duplex (IBFD) radios. This paper proposes a practical structure and presents an implementation of the ALMS loop. By employing off-the-shelf components, a prototype of the ALMS loop including two taps is implemented. The prototype is evaluated in IBFD systems which have $20 \mathrm{MHz}$ and $50 \mathrm{MHz}$ bandwidths, respectively, with the carrier frequency of $2.4 \mathrm{GHz}$. The performance of the prototype with different roll-off factors of the transmit pulse shaping filter is also examined. Experimental results show that the ALMS loop can provide $39 \mathrm{~dB}$ and $33 \mathrm{~dB}$ of SI cancellation for the two systems, respectively. Furthermore, when the roll-off factor of the pulse shaping filter changes, different levels of cancellation given by the prototype are also demonstrated accordingly. These experimental results validate the theoretical analyses presented in our previous publications on the ALMS loop behaviors.
\end{abstract}

Index Terms-In-band full-duplex, self-interference cancellation, adaptive filter, I/Q imbalance, and ALMS loop.

\section{INTRODUCTION}

The sub- $6 \mathrm{GHz}$ spectrum will be more and more congested as the Third Generation Partnership Project has indicated that the fifth-generation $(5 \mathrm{G})$ new radio systems will operate in this frequency band. In-band full-duplex (IBFD) technology which allows transceivers to simultaneously transmit and receive on a single frequency band will be extremely useful to enhance spectrum efficiency [1]. IBFD transmission is also beneficial in collision-avoidance multiple access networks by solving the problem of hidden terminals. Furthermore, this new transmission technology helps to reduce round-trip latency in multi-hop relay networks [2]. However, realization of IBFD transceivers is challenging due to its fundamental problem of self-interference (SI) as the strong signal emitted from the transmitter blocks its co-located receiver from the signal of interest. Therefore, great efforts have been devoted to mitigate the SI to a level below the noise floor of the receiver.

Self-interference can be consecutively mitigated in the propagation, radio frequency (RF) and digital domains [2]. Propagation domain methods aim to attenuate the level of SI by strategically locating transmit and receive antennas [3]-[5], or sharing one antenna by a circulator [6], [7]. Digital domain approaches generate the cancellation signal by utilizing the baseband transmitted data and channel state information to subtract the digitalized residual SI after the Analog-to-Digital converter [4]. As indicated in [8], RF domain cancellation is the most important step. Generally, RF domain approaches aim to generate a cancellation signal which mimics the SI at the input of the receiver. Some typical examples of RF cancellers are proposed in [9]-[12]. However, in most of the adaptive cancellers, digital signal processing (DSP) is required to calculate the weighting coefficients to modify the phase and amplitude of the reference signal. The involvement of DSP make these cancellers more complicated, especially in IBFD multiple-input multiple-output systems [13]. Analog least mean square (ALMS) loop proposed in [14] is a promising structure due to its simplicity and ability to achieve significant SI cancellation without any DSP. The behaviors of this adaptive filter were comprehensively investigated in [8], [14]-[18]. It has been shown that the performance of the ALMS loop depends on the transmitted signal properties and the structure of the loop. Particularly, the roll-off factor of the pulse shaping filter in the transmitter, the loop gain, the tap delay and the number of taps in the loop determine the level of cancellation given by the ALMS loop. However, these results are all confirmed by simulations in MATLAB, rather than by hardware experiments. In addition, although the ALMS loop has a simple structure, its implementation using off-theshelf components is still a challenging task. For example, the multipliers with a high conversion gain required in the loop are not available for RF signals.

In this paper, a practical structure of the ALMS loop is proposed and a prototype of the loop is implemented to provide experimental results. The prototype includes two taps for SI cancellation in IBFD systems with bandwidths of 20 $\mathrm{MHz}$ and $50 \mathrm{MHz}$ respectively at the carrier frequency of $2.4 \mathrm{GHz}$. Experimental results show that $39 \mathrm{~dB}$ and $33 \mathrm{~dB}$ of SI mitigation can be achieved by the prototype in the two systems, respectively. From the parameters of the components 
used in the prototype, the interference suppression ratio can be determined by the formula provided in [8]. Combining with the degradation factor given in [18], the practical results agree with the theoretical results. The level of cancellation is also measured with different roll-off factors of the pulse shaping filter to confirm the analyses shown in [16]. Therefore, the contributions of this paper are two fold. Firstly, it provides, for the first time, experimental results to validate the theoretical analyses in previous publications. Secondly, the implementation structure presented in this paper is proved to be useful for future applications of the ALMS loop.

The remainder of this paper is organized as follows. In Section II, the architecture and operations of the ALMS loop are described. In Section III, the design and implementation of the ALMS loop using off-the-shelf components are described. In Section IV, experimental results are presented. Finally, Section V concludes the paper.

\section{ALMS LOOP ARCHITECTURE}

The architecture of the ALMS loop proposed in [14] is depicted in Fig. 1. The ALMS loop is an adaptive filter which generates a cancellation signal to cancel the SI in the received signal at the input of the receiver. As analyzed in [7], SI channel comprises the direct and reflection paths between the transmit and receive antennas. Therefore, the ALMS loop is a multi-tap structure whose each tap includes a delay line $T_{d}$, a simple resistor-capacitor (RC) low-pass filter (LPF) and two quadrature multipliers. The delay line aims to create a delayed version of the reference signal to mimic the multipath components in the SI signal. At each tap, the amplitude and phase of the delayed reference signal are modified at the second multiplier by a weighting coefficient which is generated from the first multiplier followed by an LPF. The outputs of all the taps are combined together to generate the cancellation signal $y(t)$ which will be used to cancel the SI in the received signal $r(t)$. The residual signal $d(t)$ is amplified by the lownoise amplifier (LNA) and looped-back to the first multiplier in each tap.

To demonstrate the operations of the ALMS loop, we use the signal models as follows. Assuming the IBFD radio is a single carrier system, the modulated data symbols $a_{n}, n=$ $-\infty, \cdots, \infty$, are filtered by the root-raised cosine pulse shaping filter $g(t)$ before up-converted to RF and amplified by the power amplifier. The transmitted signal $x(t)$ at the input of the antenna is modeled as $x(t)=\operatorname{Re}\left\{X(t) e^{j 2 \pi f_{c} t}\right\}$ where $f_{c}$ is the carrier frequency, and $X(t)$ is the baseband equivalent which can be described as

$$
X(t)=\sum_{n=-\infty}^{\infty} a_{n} V_{X} g\left(t-n T_{s}\right)
$$

where $T_{s}$ is the symbol interval and $V_{X}$ is the root mean square amplitude of the transmitted signal. Noted that the pulse shaping filter is assumed to have unit power, i.e., $\frac{1}{T s} \int_{0}^{T_{s}}|g(t)|^{2} d t=1$. In addition, we assume that the transmitted data symbols $a_{n}$ are independent of each other,

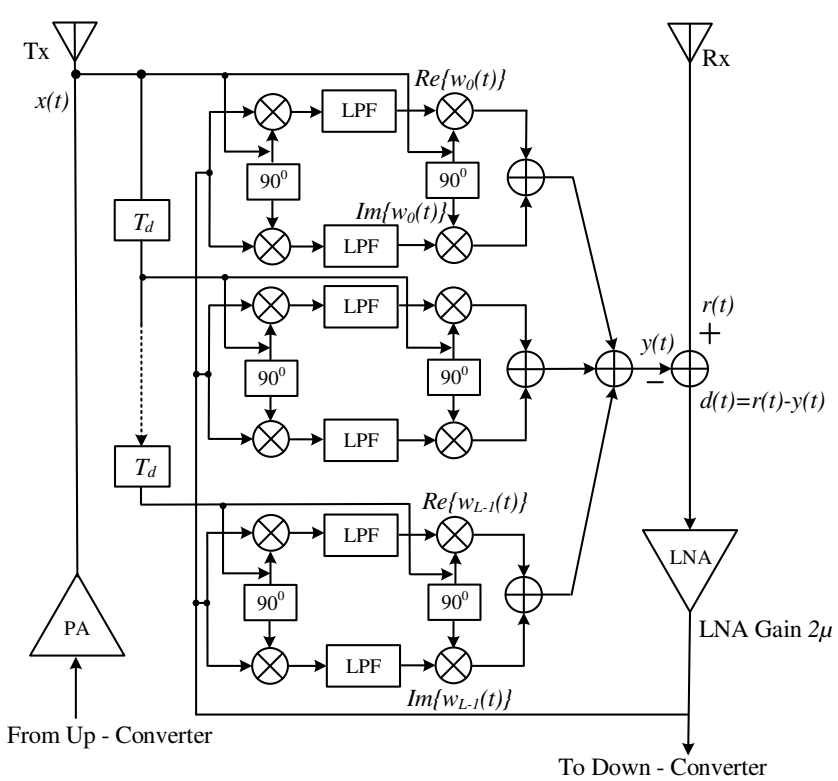

Fig. 1: The ALMS loop structure.

i.e., $E\left\{a_{n}^{*} a_{n^{\prime}}\right\}=\left\{\begin{array}{l}1, \text { for } n=n^{\prime} \\ 0, \text { for } n \neq n^{\prime}\end{array}\right.$ where $E\{$.$\} stands for$ ensemble expectation. The average power of $X(t)$ is defined as $\frac{1}{T_{s}} \int_{0}^{T_{s}} E\left\{|X(t)|^{2}\right\} d t=V_{X}^{2}$ over $1 \Omega$ load.

Due to the IBFD operation, the receive signal $r(t)$ includes the remote signal $s(t)$, the SI $z(t)$, and the additive Gaussian noise $n(t)$, i.e., $r(t)=s(t)+z(t)+n(t)$. For theoretical analysis, the SI channel is assumed to be a multi-tap filter as

$$
z(t)=\operatorname{Re}\left\{\left[\sum_{l=0}^{L-1} h_{l}^{*} X\left(t-l T_{d}\right)\right] e^{j 2 \pi f_{c} t}\right\}
$$

where $h_{l}, l=0,1, \cdots, L-1$, are the SI channel coefficients and $T_{d}$ is the delay between adjacent taps. The cancellation signal $y(t)$ is combined from the $L$ taps of the ALMS loop as

$$
y(t)=\operatorname{Re}\left\{\sum_{l=0}^{L-1} w_{l}^{*}(t) X\left(t-l T_{d}\right) e^{j 2 \pi f_{c}\left(t-l T_{d}\right)}\right\}
$$

where $w_{l}(t)$ is the complex weighting coefficient at the $l$-th tap. The residual signal $d(t)=r(t)-y(t)$ is amplified by the LNA and looped-back to all the taps of the ALMS filter. As shown in [14], the weighting coefficients $w_{l}(t)$ at the output of the LPF obtained by

$$
w_{l}(t)=\frac{2 \mu \alpha}{K_{1} K_{2}} \int_{0}^{t} e^{-\alpha(t-\tau)} d(\tau) X\left(\tau-l T_{d}\right) e^{j 2 \pi f_{c}\left(\tau-l T_{d}\right)} d \tau
$$

where $\alpha$ is the decay constant of the RC LPF ( $\alpha=1 / R C), K_{1}$ and $K_{2}$ are the dimensional constants of the first and second multipliers, respectively, and $2 \mu$ is the gain of the LNA. The dimensional constant $K_{i}$ of a multiplier is determined by its input voltages $v_{i_{1}}, v_{i_{2}}$ and its output voltage $v_{o}$ as

$$
K_{i}=\frac{v_{i_{1}} v_{i_{2}}}{v_{o}}
$$




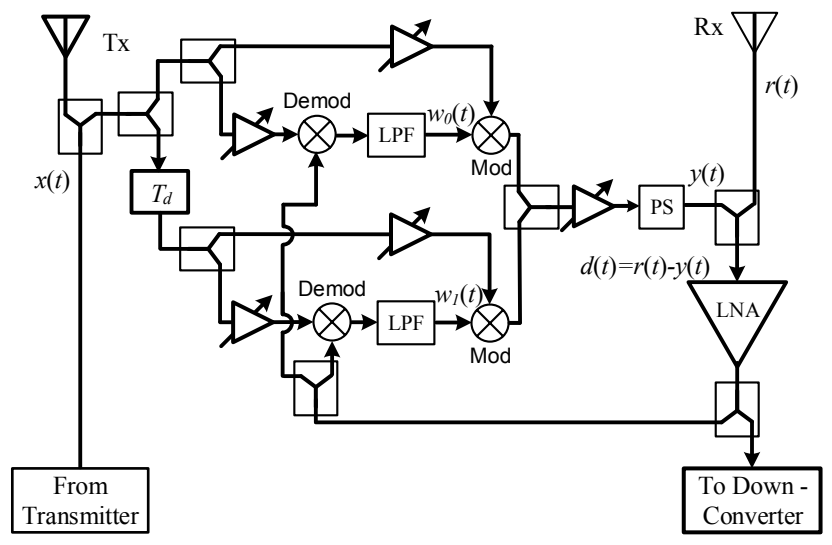

Fig. 2: A practical structure of the ALMS loop.

$i=1,2$. As analyzed in [8], when $L T_{d}$ is sufficiently large, the level of cancellation will approach the following interference suppression lower bound (ISRLB)

$$
\operatorname{ISRLB}=\frac{1+\beta(\sqrt{a+1}-1)}{(1+a)^{2}}
$$

where $a=\mu \frac{V_{X}^{2}}{K_{1} K_{2}} \frac{T_{s}}{T_{d}}$ and $\beta$ is the roll-off factor of the pulse shaping filter. Eq. (6) shows that the performance of the ALMS loop depends on the loop gain, the tap delay, the number of taps and the roll-off factor of the pulse shaping filter. Since the relationship between the tap delay $\left(T_{d}\right)$ and the number of tap $L$ is a trade-off, i.e., smaller $T_{d}$ requires larger $L$, high-gain multipliers are required to achieve lower ISRLB.

\section{IMPLEMENTATION OF ALMS LOOP}

In this section, we propose a practical structure of the ALMS loop using discrete components. Although the ALMS loop structure is simple, it is still challenging to be implemented using off-the-shelf components. In particular, the highgain quadrature multipliers in the ALMS loop are unavailable in the RF range. Therefore, instead of using these ideal multipliers, quadrature demodulators and modulators can be used. Unlike ideal multiplier which can accept any signal in its frequency range, a modulator/demodulator normally requires a single tone with a stable amplitude as a local oscillation (LO) signal. In addition, the multipliers in the ALMS loop are assumed to have a high conversion gain which is normally not applicable for modulators/demodulators. Therefore, when a modulator and demodulator are used in the ALMS loop, the reference signal should be provided to their LO ports. Then, a variable gain controlled amplifier is used at these ports to ensure the level of LO signal. The low conversion gain of the modulator/demodulator can be compensated by an amplifier after combining all the outputs of the taps.

As can be seen in Fig. 1, many power splitters are required to split and combine signals. Therefore, Wilkinson dividers are used for both splitters and combiners. The subtractor at the input of the receiver can also be implemented by a Wilkinson

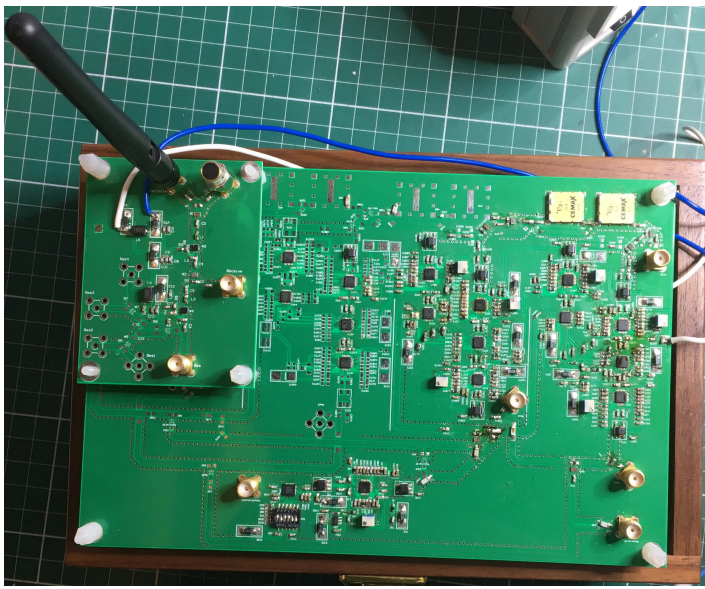

Fig. 3: Prototype of the ALMS loop and a part of the receiver.

divider with a phase shifter which make an 180 degree phase shift to the cancellation signal. However, one problem with the Wilkinson divider is that it is a lossy component. Therefore, a variable gain amplifier should be used at the input of the RF port of the demodulators to compensate this loss. The implementation structure of the 2-tap ALMS loop is presented in Fig. 2. Due to the presence of some extra components, the loop gain of this structure now can be calculated as

$$
G=\mu \frac{V_{L O}^{2}}{K_{1} K_{2}} G_{O}
$$

where $V_{L O}$ is the root-mean square amplitude of the reference signal at the LO port of the modulators and demodulators and $G_{O}$ is the power gain of the amplifier at the output of the cancellation circuit after compensating the losses of the phase shifter and the power combiner. Therefore, the interference suppression ratio, denoted as $I S R$, given by the prototype can be expected to be

$$
I S R \leq \frac{\left(1+a^{\prime}\right)^{2}}{1+\beta\left(\sqrt{a^{\prime}+1}-1\right)}
$$

where $a^{\prime}=G \frac{T_{s}}{T_{d}}$. Note that $I S R$ here is defined as the ratio between the power of SI signal without cancellation and that of the residual SI after cancellation.

The prototype of the ALMS loop is designed and fabricated on Roger 4350B printed circuit board material with all surface mount devices as shown in Fig. 3. Since this prototype is implemented to demonstrate the ALMS loop and validate the theoretical results, it is designed in a versatile form so that it can have one to four taps. The amount of delay for each tap can also be changed by cascading multiple delay lines. Therefore, the size of the prototype is $20 \mathrm{~cm} \times 13 \mathrm{~cm}$. In fact, when the ALMS loop is optimized for a specific IBFD system, its size will be much smaller. The dimension of the ALMS loop can be further minimized by manufacturing in analog integrated circuits (IC).

Detailed descriptions on components of the ALMS loop are provided as follows. The delay line of the second tap is chosen as $T_{d}=4 \mathrm{~ns}$ (DL4 RN2 Technologies). The demodulator 
(Analog Devices ADL5382) and the modulator (ADL5373) are selected for the first and the second multipliers, respectively, because they both are quadrature structure with differential output/input for the ease of interfacing. In addition, these components have a very small level of I/Q imbalances which are less than 0.3 degree and $0.07 \mathrm{~dB}$. Therefore, according to [18], the level of cancellation will only degrade by about 0.3 dB (cf. Fig. 6 in [18]). Both ADL5382 and ADL5373 require $0 \mathrm{dBm}$ of the LO signal so that $V_{L O}$ is calculated as

$$
V_{L O}=\sqrt{2 P_{L O} R}=\sqrt{2 \times 10^{(0+17-30) / 10}}=0.3166 \mathrm{~V} \text {, }
$$

where $17 \mathrm{~dB}$ is added to convert the power with a $50 \mathrm{Ohm}$ load to that of an $1 \mathrm{Ohm}$ load. From the datasheet of the ADL5382, its conversion gain is $3.5 \mathrm{~dB}$ at $2.4 \mathrm{GHz}$ if $P_{L O}=0$ $\mathrm{dBm}$. Hence, the dimensional constant $K_{1}$ can be calculated as $K_{1}=0.3166 / 10^{(3.5 / 20)}=0.2116 \mathrm{~V}$. In case of ADL5373, the output power will be $5 \mathrm{dBm}$ if the baseband voltage input is $1.4 \mathrm{~V}$ and $P_{L O}=0 \mathrm{dBm}$. Therefore, $K_{2}$ is found as

$$
K_{2}=\frac{1.4 \times 0.3166}{\sqrt{2 \times 10^{(5+17-30) / 10}}}=0.7873 \mathrm{~V}
$$

All the power splitters/combiners are Anaren PD2328J5050S2HF which have only $0.5 \mathrm{~dB}$ insertion loss. The phase shifter MACOM MAPS-010143 is used along with a power combiner to form the subtractor. The variable gain amplifier (VGA Analog Devices ADL5330) is used since its gain can be changed by a controlled voltage which is adjusted by a potentiometer. The ADL5330 at the output of the cancellation circuit is set to have $22 \mathrm{~dB}$ gain. Due to the losses caused by the power combiner $(0.5 \mathrm{~dB})$ and the phase shifter $(4.5 \mathrm{~dB}), G_{O}$ is $17 \mathrm{~dB}$.

Fig. 3 also depicts a part of the receiver with the power combiner of the subtractor and an LNA (MACOM MAAL 011078) which can provide $22 \mathrm{~dB}$ gain at $2.4 \mathrm{GHz}$. After the LNA, a power divider is used to provide the loop-back signal and residual SI signal for measurements. Since the power splitter causes a $0.5 \mathrm{~dB}$ loss at each output, the LNA gain in the loop is $21.5 \mathrm{~dB}$, i.e., $\mu=5.9425$. From these parameters and Eq. (7), we can determine the loop gain of the prototype as $G=20.1$.

\section{Measurement Results}

\section{A. Measurement setup}

In order to evaluate the performance of the prototype, a measurement test is set as shown in Fig. 4. An arbitrary waveform generator (Keysight M8190A) is used as a transmitter. Since one channel of the M8190A has two outputs which can generate the same signals. One of them can be used for the transmitter, and the other one is used for the reference signal. In all tests, the QPSK modulated symbols are passed through the root-raised cosine pulse shaping filter and then up-converted to the $2.4 \mathrm{GHz}$ carrier frequency. The power of the transmitted signal is set at the highest level of $-7.75 \mathrm{dBm}$. A $2.4 \mathrm{GHz}$ rod antenna is connected diretly to one output of the M8190A while the other port provides the reference signal for the cancellation circuit. The receive antenna is held on a

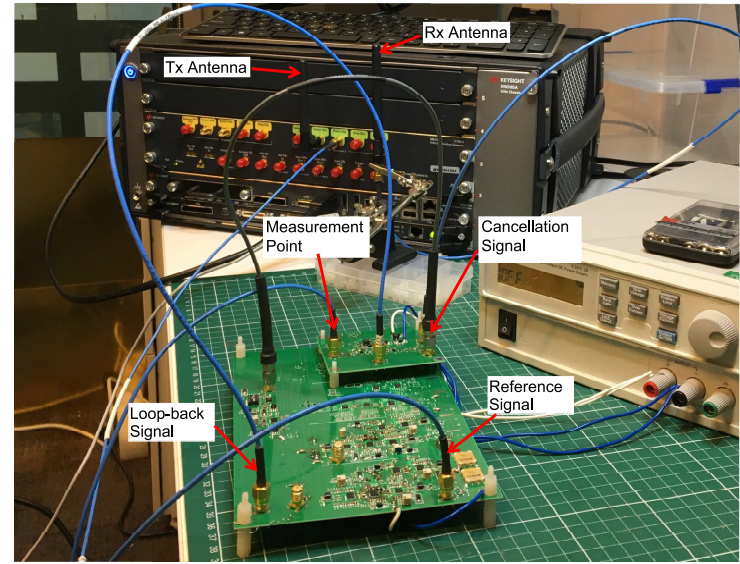

Fig. 4: The measurement setup.

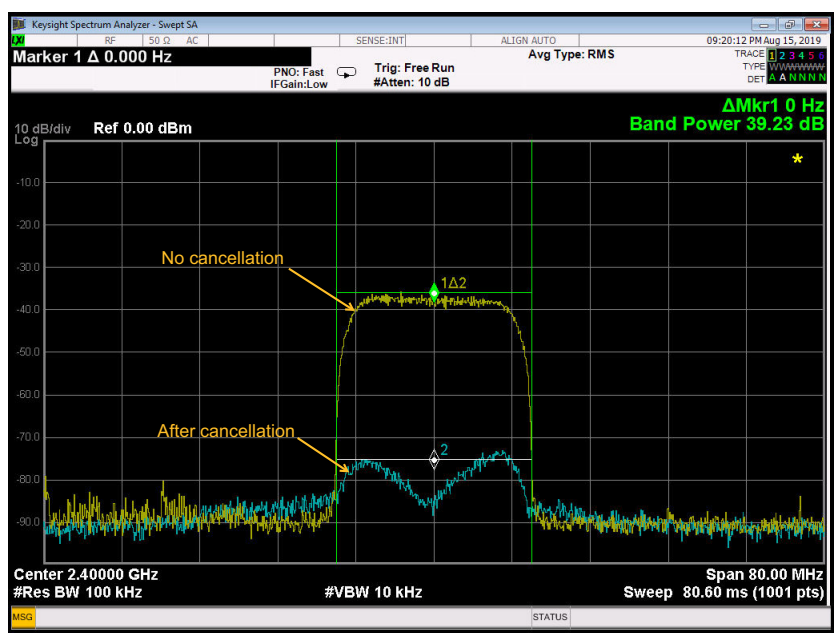

Fig. 5: Measurement results for $20 \mathrm{MHz}$ bandwidth.

holder and located at a distance of $75 \mathrm{~mm}$ to the transmit antenna. Since the cancellation circuit and the receiver are not electromagnetically shielded, they are located away from the transmitter to reduce the interference to their microstrip lines.

The signal from the receive antenna is connected to one port of the power combiner, while the cancellation signal is connected to the other port. After subtraction, the residual signal is amplified by the LNA and fed into a power splitter which provides the loop-back signal to the cancellation circuit. The other port of the power splitter is connected to the signal analyzer (Keysight PXA N9030A) for measurements.

\section{B. Measurement Results}

The first measurement is conducted with $20 \mathrm{MHz}$ transmit signal so that the data symbol period is set at $T_{s}=62.5 \mathrm{~ns}$ (i.e., $\beta=0.25$ ). Fig. 5 shows the level of cancellation given by the prototype in this case. Marker 1 indicates the difference between the $20 \mathrm{MHz}$-band power measured at $2.4 \mathrm{GHz}$ of Trace 1 and that of Trace 2. Clearly, $39.23 \mathrm{~dB}$ of cancellation is achieved by the prototype. 


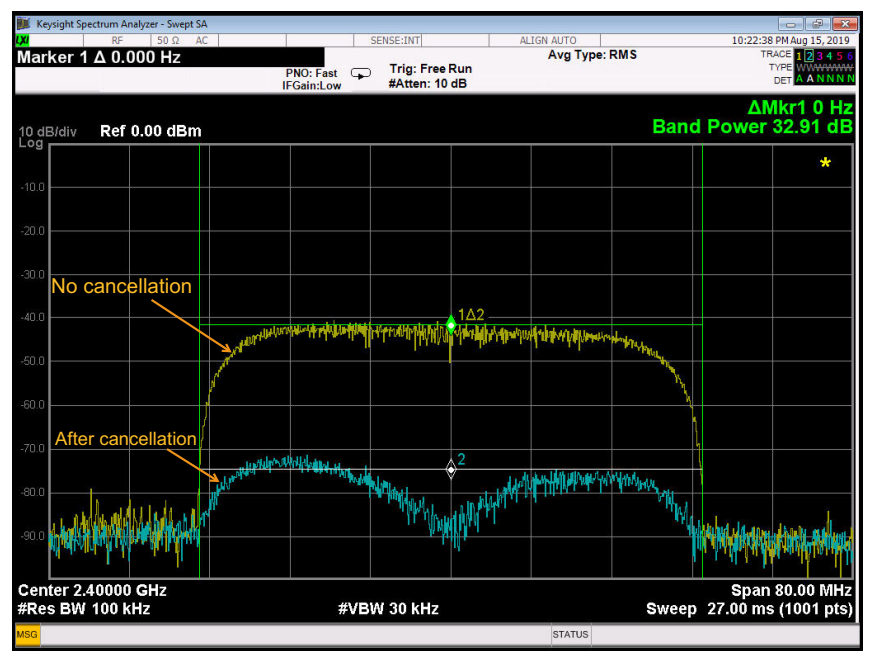

Fig. 6: Measurement results for $50 \mathrm{MHz}$ bandwidth.

In the second test, the transmitter is set to $50 \mathrm{MHz}$ bandwidth, i.e., $T_{s}=25 \mathrm{~ns}$. The results of this test are depicted in Fig. 6. We can see that $32.91 \mathrm{~dB}$ of cancellation is achieved in this case.

These experimental results are useful to validate the theoretical result presented in [8] and [18] as follows. From the symbol period of the two systems, the parameter $a^{\prime}$ is 314.06 and 125.625 in the $20 \mathrm{MHz}$ and $50 \mathrm{MHz}$ systems, respectively. Hence, the maximum level of ISR expected by the ALMS loop calculated from Eq. (8) in these two cases are 42.82 $\mathrm{dB}$ and $36.53 \mathrm{~dB}$, respectively. When I/Q imbalances of the demodulators and modulators are considered, the maximum levels of cancellation will degrade by about $0.3 \mathrm{~dB}$ [18], i.e., $42.52 \mathrm{~dB}$ and $36.33 \mathrm{~dB}$, respectively. This means that the level of cancellation in the prototype is about $3 \mathrm{~dB}$ lower than these analytical maximum levels. This is justifiable because the maximum level of cancellation can only be achieved when the number of taps and tap delay in the loop satisfy that $L T_{d}$ is very large.

In the last test, the effect of transmitted signal on the ALMS loop performance is evaluated. The roll-off factor of the pulse shaping filter in the transmitter is configured with different values while the symbol period of the transmit data is fixed at $T_{s}=62.5 \mathrm{~ns}$. Fig. 7 depicts the results of the third test. Measurement results show that the level of cancellation is $39.23 \mathrm{~dB}, 38.10 \mathrm{~dB}$, and $37.0 \mathrm{~dB}$ when the roll-off factor is $0.25,0.5$, and 0.75 , respectively. The decrease of ISR with increased roll-off factor confirms the analyses shown in [8] and [16].

\section{CONCLUSION}

This paper presents a practical structure and implementation of the ALMS loop using off-the-shelf components. The measurement results show that $39.23 \mathrm{~dB}$ and $32.91 \mathrm{~dB}$ of SI mitigations can be achieved by the prototype for IBFD systems with $20 \mathrm{MHz}$ and $50 \mathrm{MHz}$ bandwidths, respectively. The experiment with different values of roll-off factor of the

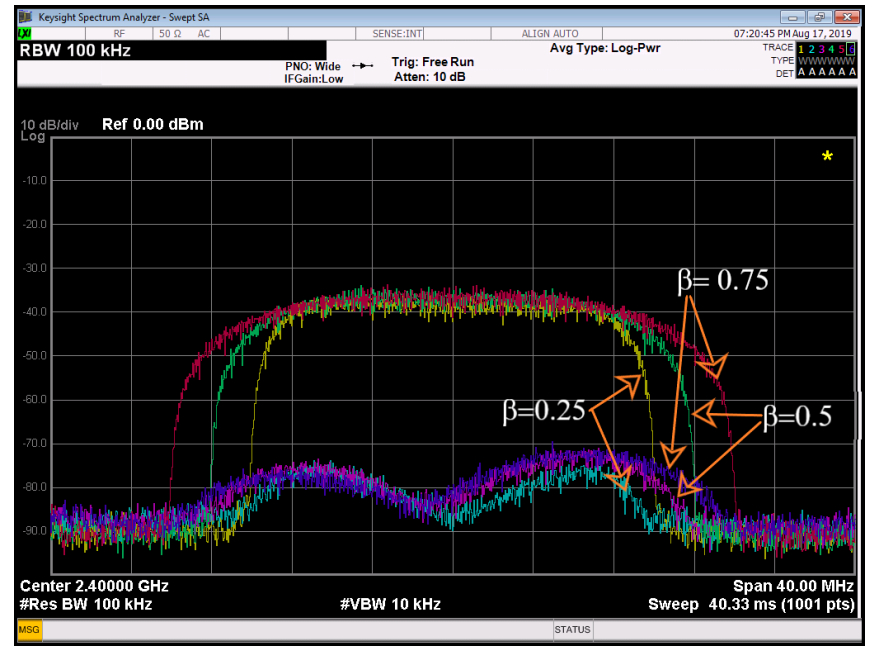

Fig. 7: Cancellation performances with different roll-off factors.

transmit pulse shaping filter also proves that the level of cancellation is affected by the transmitted signal property as analyzed in our previous publications. The proposed ALMS loop implementation structure provides a useful practical solution for future IBFD communication applications. Our future works include prototyping for IBFD systems with wider bandwidths and higher transmit powers, as well as developing a complete IBFD system.

\section{ACKNOWLEDGMENT}

This work was supported by the Australian Research Council (DP160101693).

\section{REFERENCES}

[1] K. E. Kolodziej, B. T. Perry, and J. S. Herd, "In-band full-duplex technology: Techniques and systems survey," IEEE Transactions on Microwave Theory and Techniques, vol. 67, no. 7, pp. 3025-3041, July 2019.

[2] Z. Zhang, K. Long, A. V. Vasilakos, and L. Hanzo, "Full-duplex wireless communications: Challenges, solutions, and future research directions," Proceedings of the IEEE, vol. 104, no. 7, pp. 1369-1409, July 2016.

[3] J. Choi, M. Jain, and K. Srinivasan, "Achieving single channel, full duplex wireless communication," in Proceedings of the 16th annual International Conference on Mobile Computing and Networking. Chicago, USA, Sept 2010, pp. 1-12.

[4] M. Duarte et al., "Design and characterization of a full-duplex multiantenna system for WIFI networks," IEEE Transactions on Vehicular Technology, vol. 63, no. 3, pp. 1160-1177, Mar 2014.

[5] T. Oh, Y.-G. Lim, C.-B. Chae, and Y. Lee, "Dual-polarization slot antenna with high cross-polarization discrimination for indoor small-cell mimo systems," IEEE Antennas Wireless Propagation Letters, vol. 14, pp. 374-377, Oct 2014.

[6] M. E. Knox, "Single antenna full duplex communications using a common carrier," in Proceedings of 13th IEEE Annual Wireless and Microwave Techniques Conference., (WAMICON), Florida, USA, Jun 2012, pp. 1-6.

[7] D. Bharadia, E. McMilin, and S. Katti, "Full duplex radios," in Proceedings of ACM SIGCOMM 2013, Hong Kong, China, Aug 2013, pp. 375-386.

[8] A. T. Le et al., "Frequency domain characterization and performance bounds of ALMS loop for RF self-interference cancellation," IEEE Transactions on Communications, vol. 67, no. 1, pp. 682-692, Jan. 2019. 
[9] T. Huusari et al., "Wideband self-adaptive RF cancellation circuit for full-duplex radio: Operating principle and measurements," in Proceedings 81st IEEE Vehicular Technology Conference (VTC Spring), Glasgow, Scotland, 2015, pp. 11-14.

[10] K. E. Kolodziej, J. G. McMichael, and B. T. Perry, "Multitap RF canceller for in-band full-duplex wireless communications," IEEE Transactions on Wireless Communications, vol. 15, no. 6, pp. 4321-4334, June 2016.

[11] A. Kiayani et al., "Adaptive nonlinear RF cancellation for improved isolation in simultaneous transmit-receive systems," IEEE Transactions on Microwave Theory and Techniques, vol. 66, no. 5, pp. 2299-2312, Jan. 2018.

[12] L. Zhang, M. Ma, and B. Jiao, "Design and implementation of adaptive multi-tap analog interference canceller," IEEE Transactions on Wireless Communications, vol. 18, no. 3, pp. 1698-1706, March 2019.

[13] A. T. Le, L. C. Tran, X. Huang, and Y. J. Guo, "Beam-based analog self-interference cancellation with auxiliary transmit chains in fullduplex MIMO systems," in Proceedings of 20th International Workshop on Signal Processing Advances in Wireless Communications (SPAWC), Cannes, France, 2019, pp. 1-5.
[14] X. Huang and Y. J. Guo, "Radio frequency self-interference cancellation with analog least mean-square loop," IEEE Transactions on Microwave Theory and Techniques, vol. 65, no. 9, pp. 3336-3350, Sept. 2017.

[15] A. T. Le, L. C. Tran, and X. Huang, "On performance of analog least mean square loop for self-interference cancellation in in-band full-duplex OFDM systems," in Proceedings of 85th IEEE Vehicular Technology Conference (VTC Spring), Sydney, Australia, 2017, pp. 1-5.

[16] - "Cyclostationary analysis of analog least mean square loop for self-interference cancellation in in-band full-duplex systems," IEEE Communications Letters, vol. 21, no. 12, pp. 2738-2741, Sept 2017.

[17] A. T. Le et al., "Analog least mean square loop for self-interference cancellation in generalized continuous wave SAR," in Proceedings of 88th IEEE Vehicular Technology Conference (VTC Spring), Chicago, USA, 2018, pp. 1-5.

[18] A. T. Le, L. C. Tran, X. Huang, and Y. J. Guo, "Analog least mean square loop with I/Q imbalance for self-interference cancellation in full-duplex radios," IEEE Transactions on Vehicular Technology, vol. pp, no. 99, pp. 1-13, 2019 